\title{
Mechanisms of Action Underlying the Effect of Repetitive Transcranial Magnetic Stimulation on Mood: Behavioral and Brain Imaging Studies
}

\author{
Jennifer Barrett', Valeria Della-Maggiore', Philippe A Chouinard' and Tomáš Paus*,' \\ 'Cognitive Neuroscience Unit, Montreal Neurological Institute, McGill University, Montreal, Quebec, Canada
}

\begin{abstract}
In a set of experiments, we applied $10-\mathrm{Hz}$ repetitive transcranial magnetic stimulation ( $\mathrm{rTMS}$ ) over the left mid-dorsolateral frontal cortex (MDLFC) to investigate rTMS-induced changes in affective state and neural activity in healthy volunteers. In Experiment I, we combined I0-Hz rTMS with a speech task to examine rTMS-induced changes in paralinguistic aspects of speech production, an affect-relevant behavior strongly linked to the ACC. In Experiment 2, we combined 10-Hz rTMS with positron emission tomography (PET) and used partial least squares (PLS) to identify a pattern of brain regions whose connectivity with the site of stimulation varied as a function of rTMS. The results of Experiment I revealed that following stimulation of the left MDLFC, subjects reported having less positive affect and vitality and displayed more monotonous speech. In Experiment 2, results revealed that $10-\mathrm{Hz}$ rTMS influenced the covariation between blood flow at the site of stimulation (ie the left MDLFC) and blood flow in a number of affect-relevant brain regions including the perigenual anterior cingulate gyrus, insula, thalamus, parahippocampal gyrus, and caudate nucleus. Taken together, our results suggest that changes in affect and affect-relevant behaviour following $10-\mathrm{Hz}$ rTMS applied over the left MDLFC may be related to changes in neural activity in brain regions widely implicated in affective states, including a frontocingulate circuit.

Neuropsychopharmacology (2004) 29, I 172 - I I89, advance online publication, 17 March 2004; doi: I 0. I038/sj.npp. I 3004I I
\end{abstract}

Keywords: repetitive transcranial magnetic stimulation; positron emission tomography; pitch variation; affect; functional connectivity; prefrontal cortex; anterior cingulate cortex

\section{INTRODUCTION}

Applied over the mid-dorsolateral frontal cortex (MDLFC; also referred to as the dorsolateral prefrontal cortex), repetitive transcranial magnetic stimulation (rTMS) has been shown to induce both symptom remission in depressed patients and temporary changes in affect in healthy subjects (for reviews, see George et al, 1999; Post et al, 1999; Martin et al, 2003). In depressed patients, a recent meta-analysis (Gershon et al, 2003) revealed that the most efficacious rTMS treatment protocols applied highfrequency (ie 5-20 Hz) rTMS over the left MDLFC for 5-20 days, resulting in improvement in depression ratings in $41 \%$ of the patients studied $(n=139)$. In healthy subjects, however, the short-term changes in affect observed following a single rTMS session are often opposite to the changes in mood observed in depressed patients, namely more

*Correspondence: Dr Tomáš Paus, Cognitive Neuroscience Unit, Montreal Neurological Institute, 3801 University St Montreal, QC, Canada H3A 2B4, Tel: 514398 848I, Fax: 5 I4 398 I338,

E-mail: tomas@bic.mni.mcgill.ca

Received 15 September 2003; revised 12 January 2004; accepted 14 January 2004

Online publication: 14 January 2004 at http://www.acnp.org/citations/ Npp0 I 1 60403424/default.pdf negative affect following $10-\mathrm{Hz}$ rTMS applied over the left MDLFC (eg George et al, 1996; Pascual-Leone et al, 1996; Martin et al, 1997; but for negative findings, see Mosimann et al, 2000; Padberg et al, 2001). Generally, rTMS shows promise as a novel antidepressant treatment approach as well as a tool to examine the neural substrates of emotion in healthy individuals.

The use of high-frequency rTMS applied over the left MDLFC in mood-related applications was motivated by the fact that that the lateral and medial prefrontal cortices of patients with major depression exhibit hypoperfusion and hypometabolism (for reviews of this area, see Drevets, 2000a, b; Davidson et al, 2002; Mayberg, 2003). Although studies of the motor cortex have demonstrated that highfrequency rTMS increases cortical excitability (eg Chen, 2000), the neurophysiological changes underlying rTMSinduced changes in mood are for the most part unknown. Repetitive TMS has been shown to affect neural activity at the site of stimulation as well as in distal regions believed to be connected to the site of stimulation (reviewed in Paus, 2002). Thus, one possibility is that rTMS influences mood by acting on neural circuits important in the expression and regulation of affective symptomatology. In support of this hypothesis, the MDLFC (comprised of cytoarchitectonic areas 9, 46, and 9/46 as defined by Petrides and Pandya, 
1999) is known to be richly interconnected with a number of brain regions long implicated in mood, motivation, and arousal such as the striatum, thalamus, and the anterior cingulate cortex (Petrides and Pandya, 1999; Barbas, 2000). Knowledge of how rTMS influences the connectivity and excitability of the MDLFC may contribute to our understanding of rTMS mechanisms of action in depression as well as the neural pathways regulating normal and pathological affective states.

With the aim of understanding the mechanisms by which rTMS alleviates depression, a number of investigators have combined rTMS treatment with brain imaging. In a typical protocol, depressed patients are scanned with single photon emission computed tomography (SPECT) or positron emission tomography (PET) before and after rTMS applied over the left MDLFC (1-20 Hz frequency, 1-10 sessions, MDLFC defined as $5 \mathrm{~cm}$ anterior to the hand area of the primary motor cortex, eg Kimbrell et al, 1999; Teneback et al, 1999; Speer et al, 2000; Catafau et al, 2001; Nahas et al, 2001; Conca et al, 2002; Mottaghy et al, 2002; Nadeau et al, 2002; Shajahan et al, 2002). Following a single session of high-frequency rTMS, Shajahan et al (2002) reported that the connectivity between a number of brain circuits implicated in affect was significantly stronger. Following 5-10 days of rTMS treatment applied over the left MDLFC, a number of investigators have also found changes in neural activity in the left prefrontal cortex (Speer et al, 2000; Catafau et al, 2001; Nahas et al, 2001) as well as in affectrelevant brain regions, including the anterior cingulate cortex (ACC), amygdala, and striatum (Teneback et al, 1999; Speer et al, 2000; Nahas et al, 2001; Nadeau et al, 2002). Finally, as with antidepressant drugs (eg Mayberg et al, 1997), baseline resting cerebral blood flow and cerebral glucose metabolism values in the ACC have been found to be predictive of antidepressant response to rTMS treatment (Kimbrell et al, 1999; Mottaghy et al, 2002).

Although this body of work provides some information about the brain regions where changes in neural activity may underlie the 'antidepressant effect' of rTMS or the brain regions where neural activity predicts an rTMS treatment response, a number of methodological issues make it difficult to discern the effect of rTMS applied over the MDLFC on brain activity. For example, the majority of previous studies imaged the brains of depressed patients during resting state, a practice that does not control for the variable cognitive processes that patients may be engaged in during scanning. In addition, an especially challenging and often unavoidable aspect of imaging depressed patients is the commonly observed heterogeneity in terms of both symptom expression and medications, which likely influence baseline blood flow levels and possibly, the location and direction of rTMS-induced changes in neural activity. In light of these issues, assessing rTMS acute effects in healthy subjects could help elucidate the changes in brain activity observed following chronic rTMS treatment.

To this aim, in a previous study (Paus et al, 2001), we applied $10-\mathrm{Hz}$ rTMS over the left MDLFC of healthy volunteers and examined the connectivity of this region with PET. In our paradigm, rTMS was applied between scans, as 'conditioning' stimulation. In addition, to standardize the scanning environment and to 'probe' for rTMS-induced changes in brain activity, double-pulse low- frequency $(0.5 \mathrm{~Hz})$ TMS was also applied during scans. Our results supported the existence of strong connections between the MDLFC and the ACC, possibly implicating the modulation of a frontocingulate circuit in the antidepressant effect of rTMS. This finding was particularly relevant as the ACC is believed to play a strong role in both the pathophysiology of major depression as well as the neural pathways underlying normal affect (Devinsky et al, 1995; Bush et al, 2000; Drevets, 2001; Paus, 2001; Davidson et al, 2002; Mayberg, 2003).

The goal of the current study was to investigate further, in healthy volunteers, the neural substrates of $10-\mathrm{Hz}$ rTMSinduced changes in affective state. In Experiment 1, we combined $10-\mathrm{Hz}$ rTMS applied over the left MDLFC with a speech task to examine rTMS-induced changes in an affectrelevant behavior, namely paralinguistic aspects of speech production. In our previous work, we described a robust effect of a 10-min mood induction on paralinguistic aspects of speech, including variation in pitch and loudness (Barrett and Paus, 2002), and confirmed the role of the ACC in mediating this phenomenon (Barrett, Pike and Paus, 2004). Based on our previous studies and past rTMS investigations in healthy subjects, we predicted that $10-\mathrm{Hz}$ rTMS applied over the left MDLFC would decrease affect and measures of speech pitch and loudness. In Experiment 2, we combined $10-\mathrm{Hz}$ rTMS applied over the left MDLFC with PET and used PLS to identify brain regions whose connectivity with the site of stimulation varied as a function of rTMS. We expected that, complementary to the cerebral blood flow (CBF) increases observed in our past study (Paus et al, 2001), the connectivity between the left MDLFC and the ACC may vary as function of $10-\mathrm{Hz}$ rTMS. As a control, we also examined the effect of $1-\mathrm{Hz}$ rTMS on both brain activity and behavior: low-frequency rTMS (ie $0.5-1 \mathrm{~Hz}$ ) has been associated with decreased cortical excitability (eg Chen, 2000).

\section{MATERIALS AND METHODS}

\section{Experiment 1: The Effect of 10-Hz rTMS on Affect and Speech}

Subjects. A total of 10 healthy right-handed women (age range $20-26$ years; $10-\mathrm{Hz}$ Group, $n=5 ; 1-\mathrm{Hz}$ Group $n=5$ ) with no personal history of neurological or psychiatric disorders were studied; all were students of McGill University. With the exception of oral contraceptives (10$\mathrm{Hz}$ Group, $n=4$; $1-\mathrm{Hz}$ Group, $n=4)$, subjects were not taking any prescribed or over-the-counter medications. To screen for past or present mood difficulties, subjects were administered the Mood Disorder module of the Structured Clinical Interview for DSM-IV Axis I Disorders (SCID; First et al, 2002) by the first author. In addition to the Mood Disorder module, the SCID screening questionnaire for all major psychiatric disorders and conditions (eg anxiety disorder, substance abuse, eating disorders, schizophrenia) was also administered. Subjects were excluded from the study if they responded positively to any of the screening questions. All experimental protocols were approved by the Research Ethics Board of the Montreal Neurological Institute and Hospital and all subjects provided written informed consent prior to participating. 
Experimental design. Subjects completed two rTMS sessions that were held on consecutive days. Each subject completed both sessions at the same time of day. In each session, three 2.5-min series of rTMS were applied over the MDLFC. The two sessions differed only in the side of stimulation (left $v s$ right); session order was counterbalanced. For the left hemisphere, we used the same stimulation site $(x=-40, y=32, z=30$, corresponding to Brodmann area 9 and cytoarchitectonic area $9 / 46$ as defined by Petrides and Pandya, 1999) as employed in our previous TMS-PET studies (Paus et al, 2001; Strafella et al, 2001). It is of note that this brain region was found to overlap with the brain region targeted by the 'standard procedure' for placing the coil over the MDLFC, which involves finding the hand area of the primary motor cortex and moving the coil $5 \mathrm{~cm}$ rostral to this location (Rektorova and Paus, unpublished data). The site of stimulation for the right hemisphere was $x=40, y=32$ and $z=30$.

One group of subjects received rTMS at $10-\mathrm{Hz}$ frequency $(10-\mathrm{Hz}$ Group) and another received rTMS at $1-\mathrm{Hz}$ frequency $(1-\mathrm{Hz}$ Group). To probe for rTMS-induced changes in affect and behavior before and after stimulation, subjects completed affect questionnaires and a speech task. As the left MDLFC has been previously implicated in working memory (eg Petrides et al, 1993), subjects also completed a verbal working memory task. The study was described as an investigation of rTMS effects on information processing and all subjects were naïve to the experimental aims of the study. The two groups of subjects were tested two months apart $(10-\mathrm{Hz}$ Group, then $1-\mathrm{Hz}$ Group).

rTMS prescreening. TMS was applied using the Cadwell high-speed magnetic stimulator and a circular coil (external diameter, $9 \mathrm{~cm}$ ). At the start of each session, resting motor threshold (MT) was determined in each subject by applying single-pulse TMS to the motor cortex and determining the minimum intensity necessary to elicit motor-evoked potentials in the first interosseus muscle of the contralateral hand. Subjects with an MT greater than $80 \%$ of the maximum stimulator output were excluded from the study; this was a technical limitation related to the overheating of the coil during rTMS applied at high intensities. When the left MDLFC was stimulated, the MT was determined for the left hemisphere; when the right MDLFC was stimulated, the MT was determined for the right hemisphere. No significant differences were found when comparing subject thresholds between hemispheres and groups.

rTMS procedure. To target the same cortical location in all subjects, we used a procedure developed in our first TMSPET study (for details, see Paus et al, 1997; Paus, 2002), which takes advantage of both standardized stereotaxic space and frameless stereotaxy. In short, MR images (160 continuous 1-mm thick saggital slices) of the subject's brain were acquired and the MDLFC coordinates in MNI space were transformed to the subject's brain coordinate (ie 'native') space. The subject's MDLFC coordinates were then 'marked' on the MR image. Next, with the subject lying down, the coil was positioned over the target location with the use of frameless stereotaxy. Accurate placement of the coil was achieved by tracking the coil position and three- dimensional orientation with an infrared optical tracking system (Polaris System, Northern Digital, Waterloo, ON, Canada and Brainsight software, Rogue Research Inc., Montreal, QC, Canada). When the location was reached, it was marked on the subject's head and the coil was locked into place using a custom-designed coil holder. The coil was positioned so that the anterior tip of the coil was closest to the target location and the rest of the coil was tilted away from the subject's skull; the induced current under to coil flowed in a lateromedial direction (same coil placement as in Strafella et al, 2001).

Three 2.5-min series of rTMS were applied at $100 \%$ MT. In the $10-\mathrm{Hz}$ Group, each 2.5-min series consisted of 151 -s trains, with a 10-s intertrain interval. In the $1-\mathrm{Hz}$ Group, each $2.5 \mathrm{~min}$ series consisted of one 150-s train. After the first and second series of rTMS, 10-min breaks were given, at this time the coil was removed from over the subject's head and cooled with an ice pack. The marked scalp location was used to reposition the coil throughout the study. During the stimulation and breaks, subjects were lying down with their eyes closed on a fully reclined chair and wore earplugs to protect their hearing. The same two experimenters (JB and $\mathrm{PC}$ ) were always in the room with the subject for the rTMS portion of the experiment, including breaks. After every rTMS series, an experimenter (JB) ensured that the subject was not experiencing any discomfort using questions that were standardized across subjects. In addition, an experimenter (JB) alerted the subject to the start of the next rTMS series following the break period. This was the only communication between the subject and the experimenters throughout the rTMS procedure.

Speech task and working memory task. Immediately before rTMS and $5 \mathrm{~min}$ after the last series of rTMS, subjects were left alone in the laboratory room to complete a speech task and a working memory task; the order of task presentation was counterbalanced. A 5-minute break was given between the last rTMS series and subsequent testing as in our previous TMS-PET study (Paus et al, 2001), the maximum changes in $\mathrm{CBF}$ in the ACC and MDLFC were observed $10 \mathrm{~min}$ after the last series of $10-\mathrm{Hz}$ rTMS. Prior to the experiment in both sessions, subjects completed a practice session to become familiarized with the tasks. The presentation of all stimuli was controlled by SuperLab Pro software (SuperLab Pro, v. 2.0 2000; Cedrus, San Pedro, Calif., USA) installed on a personal computer. Subject responses were recorded through a serial-port mouse.

For the speech task, subjects read emotionally neutral sentences out loud and at their own pace. Sentence stimuli were chosen from the Revised Harvard Psychoacoustic Sentences (IEEE Standards, 1969) and were comprised of four 20-sentence sets where segmental phonemes of the English language are represented in accordance with their frequency of use. Different sentence sets were read before and after rTMS. In addition, different sentence sets were read in each session (left MDLFC, right MDLFC); the order of sentence set presentation was counterbalanced. During the task, one sentence was presented on the computer screen at a time. Subjects were required to read the sentence out loud and press the left mouse button to end the trial. 
Speech was recorded using a noise-reducing 'head set' microphone, and stored on a personal computer using CoolEdit 2000 (Syntrillium Software, Phoenix, AZ, USA). The distance of the head-set microphone from the subject's mouth was held constant before and after rTMS. For off-line analysis of the speech samples, a Matlab ${ }^{\circledR}$ (Mathworks Inc., Natick, MA, USA) platform was adapted to extract the following parameters from the sentences: range of amplitude, root-mean-square (RMS) amplitude, mean fundamental frequency $\left(F_{0}\right)$, standard deviation of $F_{0}$ and range of $F_{0}$. RMS amplitude is an acoustical correlate of loudness, and measuring the range of amplitude provides information about the variability of loudness throughout an utterance. Fundamental frequency, the dominating frequency of the sound produced by the vibration of the vocal folds, is a major contributor to perceived vocal pitch; $F_{0}$ variation across a speech sample reflects the amount of intonation in speech. Detailed information regarding our speech analysis procedure has been previously reported (Barrett and Paus, 2002). For both groups and for both hemispheres and testing sessions (pre-rTMS, post-rTMS), subject means (ie the mean of the 20 sentences) were determined for each of the speech parameters.

Subjects also completed a verbal working memory task (Petrides et al, 2001); a set of five words were used as stimuli (brave, force, style, modal, exact). During each trial, four out of the five words were presented successively in random order at the center of a computer screen and the subject was required to monitor their occurrence. After the presentation of the fourth word, a delay of one second occurred after which a target word was presented. The subject had to indicate whether the target word was one of the four items presented prior to the delay or whether it was the item from the set of five that had not been presented. Responses were indicated by pressing a mouse button (yes = right button, no = left button). The subject had $1.5 \mathrm{~s}$ to respond after which a new trial began; there were a total of 24 trials. For both groups and for both hemispheres and testing sessions (pre-rTMS, post-rTMS), subject means were determined for response time (ie the time from the presentation of the target word and the response) and accuracy (ie percent correct responses).

Affect questionnaires. Immediately before rTMS and $5 \mathrm{~min}$ after the last series of rTMS, subjects also completed an affect questionnaire, the Positive and Negative Affect Scale (PANAS; Watson et al, 1988) and the Vitality Scale (Ryan and Frederick, 1997).

The affect questionnaire assessed levels of comfort, fatigue, irritation, mood, anxiety, and pain. Ratings were made on a seven-point Likert scale, with -3 indicating the highest negative level and +3 indicating the highest positive level for each affective state. For example, the mood rating ranged from I feel very sad (-3) to I feel very happy $(+3)$ and the fatigue rating ranged from I feel very fatigued $(-3)$ to I feel very rested $(+3)$. For both groups and for both hemispheres and testing sessions (pre-rTMS, post-rTMS), subject means were determined for each of the six affect scores, as well as for the mean of the six scores.

The PANAS is a 20 -item scale that asks subjects to rate their level of positive affect (eg 'interested', 'strong', number of items $=10$ ) and negative affect (eg 'distressed', 'ashamed', number of items $=10$ ). Ratings were made on a five-point scale, with a rating of 1 indicating a low level of a given affect ('very slightly or not at all') and a rating of 5 indicating a high level of a given affect ('extremely'). For both groups and for both hemispheres and testing sessions (pre-rTMS, post-rTMS), subject means were determined separately for positive and negative affect.

The Vitality Scale is a seven-item scale that measures the subjective feeling of being 'alive' and alert, in other words, to have energy available to the self. Ratings on the seven items (eg 'At this time, I have energy and spirit') were made on a seven-point scale ranging from 1 ('not at all true') to 7 ('very true'). For both groups and for both hemispheres and testing sessions (pre-rTMS, post-rTMS), subject means were determined for the seven scale items.

Statistical analysis. To compare the effects of rTMS on speech production, affect, and working memory, we conducted an analysis of variance (ANOVA) with hemisphere of stimulation (left hemisphere, right hemisphere) and testing session (pre-rTMS, post-rTMS) as withinsubjects factors, separately for the $10-$ and $1-\mathrm{Hz}$ groups. In all analyses, the order in which the hemispheres were stimulated (ie left hemisphere stimulated during session 1 or right hemisphere stimulated during session 1) was used as a covariate.

\section{Experiment 2: rTMS Effects on Brain Activity Measured by Regional Cerebral Blood Flow}

Subjects. A different set of eight healthy right-handed women (age range 19-26 years) with no personal history of neurological or psychiatric disorders were studied: all were students of McGill University and none participated in Experiment 1. As in Experiment 1, subjects were not taking any prescribed or over-the-counter medication other than oral contraceptives $(n=5)$. Subjects were screened for past or present mood difficulties in the same manner as Experiment 1. All experimental protocols were approved by the Research Ethics Board of the Montreal Neurological Institute and Hospital and all subjects provided written informed consent prior to participating.

Experimental design. Each subject completed two TMSPET sessions that were held on consecutive days; session order was counterbalanced and each subject completed both sessions at the same time of day. We used the same stimulation site $(x=-40, y=32, z=30)$ as described in Experiment 1 . In each session, six PET scans were acquired. Before the third, fourth, and fifth scans, a 2.5-min series of 'conditioning' rTMS was applied over the left MDLFC (see Figure 1). To probe for changes in connectivity and excitability before and after rTMS, rTMS was also applied during the second, third, fourth, fifth, and sixth scans; the first scan was a baseline scan with no rTMS applied. The two sessions differed in the frequency of 'conditioning' rTMS administered between the scans $(1 \mathrm{~Hz} v s 10 \mathrm{~Hz})$ and in the frequency of rTMS administered during the 'probe' scans; $10-\mathrm{Hz}$ rTMS was applied during the probe scans following $1-\mathrm{Hz}$ rTMS, and $1-\mathrm{Hz}$ rTMS was applied during the probe scans following $10-\mathrm{Hz}$ rTMS. We expected that, 


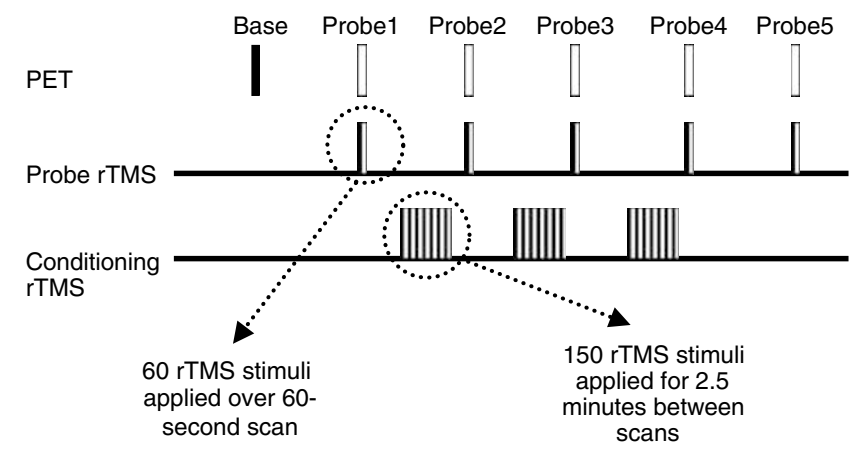

Figure I Design of Experiment 2. Each subject completed two TMSPET sessions that were held on consecutive days. In each session, six PET scans were acquired. Before the third, fourth, and fifth scans, a 2.5-min series of 'conditioning' rTMS was applied over the left MDLFC. To probe for changes in connectivity and excitability before and after rTMS, rTMS was also applied during the second, third, fourth, fifth, and sixth scans; the first scan was a baseline scan with no rTMS applied. The two sessions differed only in the frequency of 'conditioning' rTMS administered between the scans ( $\mathrm{I}$ vs $\mathrm{IOHz}$ ) and in the frequency of rTMS administered during the 'probe' scans; $10-\mathrm{Hz}$ rTMS was applied during the probe scans following $\mathrm{I}-\mathrm{Hz}$ rTMS and $\mathrm{I}-\mathrm{Hz}$ rTMS was applied during the probe scans following $10-\mathrm{Hz}$ rTMS.

consistent with our previous observations and with the posited effects of $10-\mathrm{Hz}$ vs $1-\mathrm{Hz}$ rTMS on cortical excitability (Paus et al, 2001; Chen, 2000), 10-Hz conditioning rTMS would 'reverse' $1-\mathrm{Hz}$ rTMS-induced decreases in CBF during the probe scans. Further, we also hypothesized that $1-\mathrm{Hz}$ conditioning rTMS may 'reverse' $10-\mathrm{Hz}$ rTMSinduced increases in CBF during the probe scans.

rTMS prescreening. TMS was applied using the Cadwell high-speed magnetic stimulator and a figure-of-eight coil (Corticoil, 5-cm inner diameter of one wing). Approximately 1 week prior to the TMS-PET session, resting MT was determined as in Experiment 1. In addition, subjects with an MT greater than $80 \%$ of the maximum stimulator output were again excluded from the study.

Coil positioning. To target the same cortical location in all subjects, we used the frameless-stereotaxy procedure described in Experiment 1. When the location was reached, the coil was locked into place using a custom-designed coil holder.

TMS-PET session. To protect the photomultipliers in the PET detectors from the effects of the coil-generated magnetic field, a well-grounded cylindrical insert consisting of four layers of $0.5-\mathrm{mm}$-thick mu metal was placed in the scanner's patient port prior to the start of the session. Subjects were placed in the scanner and the coil was positioned over the left MDLFC. To minimize head movement during the session, subjects were fit with a customized mouth piece (ie 'bite-bar'). Earplugs were worn to protect hearing.

Following the insertion of a catheter into the left antecubital vein, a 10-min transmission scan was acquired. Data from the transmission scan were used to correct for the attenuation of $\gamma$-rays due to all objects in the scanner, including the coil, the coil mount, and the metal insert. The transmission scan also permitted verification of the coil position; this procedure will be described in greater detail below.

Six PET scans were obtained with a CTI/Siemens $\mathrm{HR}^{+}$ 63-slice tomograph operating in 3-D acquisition mode. The distribution of CBF was estimated during a 60-s scan by using $\left[{ }^{15} \mathrm{O}\right] \mathrm{H}_{2} \mathrm{O}$. In each scan, a bolus of $10 \mathrm{mCi}\left[{ }^{15} \mathrm{O}\right] \mathrm{H}_{2} \mathrm{O}$ was injected through the catheter.

For both sessions, rTMS was applied at $100 \%$ of resting MT. In the ' $10-\mathrm{Hz}$ Conditioning' session, a 2.5 -min series of 10-Hz rTMS (15 1-s trains; 10-s between trains) was applied before the third, fourth, and fifth scans and a 60-s train of $1-\mathrm{Hz}$ rTMS was applied during the second, third, fourth, fifth, and sixth scans (ie the probe scans). In the ' $1-\mathrm{Hz}$ Conditioning' session, a 2.5-min series of $1-\mathrm{Hz}$ rTMS (one 150-s train) was applied before the third, fourth and fifth scans, and a 1-min series of 10-Hz rTMS (six 1-s trains, 10-s between trains) was applied during the second, third, fourth, fifth, and sixth scans (see Figure 1). No rTMS was administered during the first scan (baseline scan). The order of the baseline scan and probe scan 2 (ie the probe scan before first series of conditioning rTMS) was counterbalanced. During all scans and during conditioning rTMS, subjects were instructed to remain relaxed with their eyes closed. Further, a custom-designed air-cooling system was used to prevent the coil from becoming uncomfortably warm during the stimulation.

Affect questionnaire. Before and after the TMS-PET session, subjects completed the same affect questionnaire as described in Experiment 1. For both rTMS sessions (10$\mathrm{Hz}$ Conditioning, $1-\mathrm{Hz}$ Conditioning) and testing points (pre rTMS, post rTMS), subject means were determined for each of the six affect scores, as well as for the mean of the six scores.

Verification of coil position. The PET transmission scan was used to verify the final position of the coil relative to the acquired PET and MRI images in each subject. The transmission image of the coil was registered with its $\mathrm{X}$-ray image, and a straight rod orthogonal to the plane of the coil was projected from the coil center (Paus and Wolforth, 1998). Following PET-to-PET, PET-to-MRI, and MRI-Talairach transformations, the location of the rod indicating the projected center of the coil in each of the eight subjects were superimposed on an average MRI in standardized stereotaxic space (see Figure 2a).

\section{Statistical analysis of PET images.}

Image preprocessing: PET images were preprocessed by realigning all images obtained in each subject to their first image, spatially transforming the images into the standard stereotaxic space of Talairach and Tournoux (1988) and spatial smoothing using a $10-\mathrm{mm}$ isotropic Gaussian filter. All preprocessing steps for the PLS analyses were performed with Statistical Parametric Mapping software (SPM99, Wellcome Department of Cognitive Neurology, London). In addition, to control for individual differences in wholebrain CBF, subject scans were adjusted for global bloodflow values by dividing each voxel value by the average whole-brain blood flow value within a scan. 

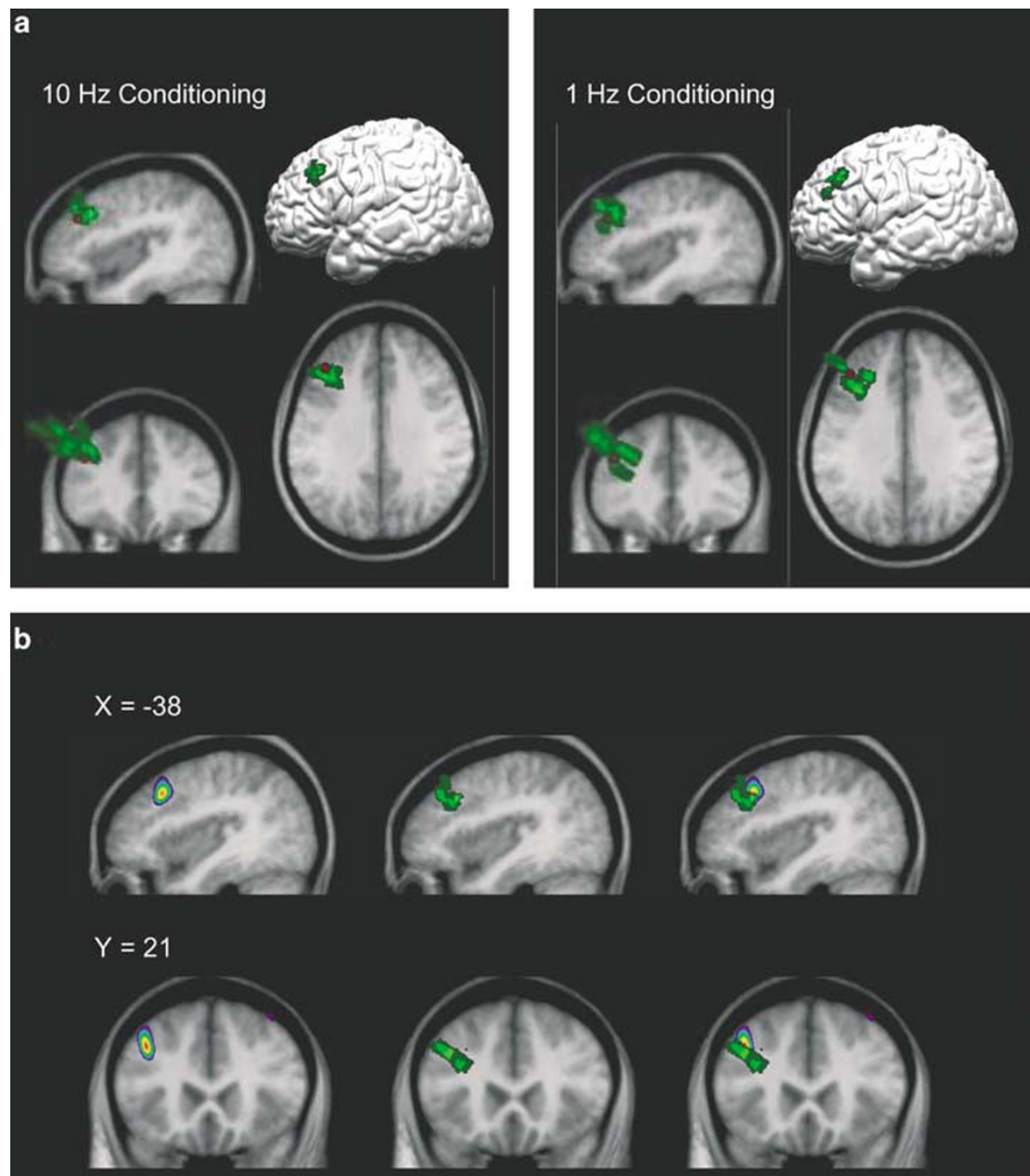

\section{$Z=37$}
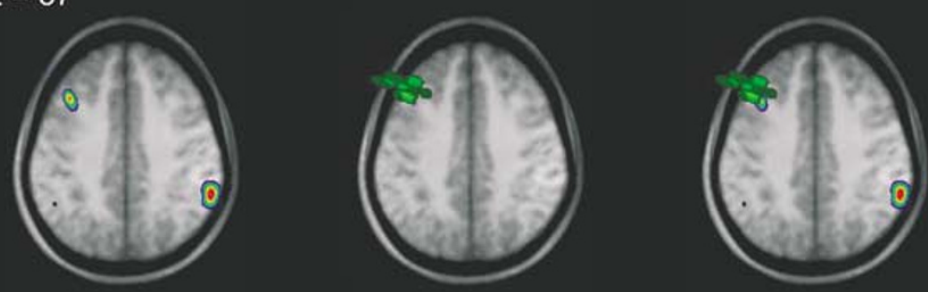

Figure 2 (a) Verification of TMS coil placement. In Experiment 2, the PET transmission scan was used to verify the final position of the coil relative to the acquired PET and MRI images in each subject. The transmission-scan image of the coil was registered with its $\mathrm{X}$-ray image, and a straight rod orthogonal to the plane of the coil was projected from the coil center (Paus and Wolforth, 1998). Following PET-to-PET, PET-to-MRI, and MRI-Talaraich transformations, the location of the rod indicating the projected center of the coil in each of the eight subjects were superimposed on an average MRI in standardized stereotaxic space. (a) Contains the centre of the coil for each subject (represented in green) and the target location $(x=-40, y=32, z=30$; represented in red). (b) Significant change in CBF at the site of stimulation. For the $10-\mathrm{Hz}$ Conditioning session, a significant increase in CBF was observed in the left MDLFC at the site of stimulation $(x=-38, y=21, z=37, t=3.82)$ when subtracting the average of the Probes 2,3 and 4 (ie the scans immediately following the between-scan stimulation) from Probe 5. The first column of (b) contains the $x, y$, and $z$ locations for the CBF peak at the site of stimulation ( $t=3.83$; $p<0.0002$, two-tailed, uncorrected); the second column of (b) contains the $x, y$, and $z$ locations for the average coil placement (ie average location of center of TMS coil); the third column of (b) contains the overlap of the peak location and the average coil placement.

Partial least squares: A full description of partial least squares (PLS) can be found in McIntosh et al (1996a). Based on the covariance between $\mathrm{CBF}$ at each voxel and $\mathrm{CBF}$ at the site of stimulation, seed-voxel PLS extracts latent variables that best reflect the relationship between the $\mathrm{CBF}$ at the site of interest (seed voxel) and CBF in the rest of the brain. This technique has been used to explore specific hypotheses concerning the functional connectivity of regions of interest 
(eg McIntosh et al, 1996; Della-Maggiore et al, 2000). In the present study, we used seed-voxel PLS to identify a pattern of brain regions whose CBF varied as a function of rTMS. The procedure involved three steps.

First, the correlation between CBF values in the left MDLFC (ie the 'seed', see below) and each voxel were computed for each scan across subjects. This produced one correlation map per scan; all 42 maps (six scans, seven subjects) were entered into one matrix. Second, the singular value decomposition of this matrix produced six (one per scan) mutually orthogonal latent variables (LVs) consisting of a singular image and a scan profile. Singular images contained a weighted linear combination of voxels that covaried with CBF in left MDLFC. The numerical weights within the images are called saliences and can be positive or negative. Third, each singular image was then multiplied (dot product) by each subject's raw image, yielding individual brain scores. The correlation between CBF for MDLFC and the brain scores for each scan produced scan profiles that aid in interpreting the contribution of each scan to each singular image. Positive saliences in the singular image correlate positively with the singular profile, whereas negative saliences correlate negatively with the singular profile. If, for example, the singular profile is similar across scans, salient areas in the singular image would relate similarly with CBF in the MDLFC as a function of rTMS. Conversely, if the singular profile differs between scans, then the singular image would reflect a timedependent effect of rTMS on the relationship between CBF in MDLFC and functionally associated regions.

To assess whether or not the patterns represented by each LV were significant, a permutation test was executed (McIntosh and Gonzalez-Lima, 1998). In addition, the reliability of voxel saliences (analogous to $Z$-values) in the singular image was assessed by bootstrap estimation of the standard error. In interpreting voxel saliences within the singular image, voxels were considered reliable if they had a ratio of salience to SE greater than three (Efron and Tibshirani, 1986).

Determination of the seed voxel: To identify a 'seed' voxel, $\mathrm{CBF}$ images were first reconstructed with a $10-\mathrm{mm}$ Hanning filter, normalized for differences in global CBF, coregistered with the individual MRIs (Woods et al, 1993) and transformed into stereotaxic space (Talairach and Tournoux, 1988) using an automated feature-matching algorithm (Collins et al, 1994). Next, using in-house software (DOT, available at http://www.bic.mni.mcgill.ca/ users/sylvain/doc/html/dot/index.html), subtraction maps were generated to determine if there was an rTMS-induced change in $\mathrm{CBF}$ at the site of stimulation when comparing the following contrasts: (1) Base and Probe 1, (2) the average of Probes 2, 3, and 4 (ie the scans immediately following conditioning rTMS) minus Probe 1 and (3) Probe 5 minus the average of Probes 2, 3, and 4 (see Figure 1). To assess the significance of each contrast, $t$-values were calculated at each voxel by dividing the mean CBF difference by its standard deviation pooled across all voxels (Worsley et al, 1992). Significant $t$-values at the site of stimulation were determined using a directed search, where we considered values equal to or exceeding a criterion of $t=3.5$ as significant ( $p<0.0002$, two-tailed, uncorrected).

\section{RESULTS}

\section{Experiment 1: rTMS Effects on Affect and Speech}

All means and standard deviations (adjusted for the order in which the hemispheres were stimulated) are presented in Table 1. For each dependent measure, we conducted an analysis of variance (ANOVA) with hemisphere of stimulation (left, right) and testing session (pre-rTMS, post-rTMS) as within-subject factors and the order of hemisphere stimulation (left hemisphere first, right hemisphere first) as a covariate. It is of note that during debriefing with the experimenter, there were no systematic reports of subjective differences between left and right MDLFC stimulation, for either the $10-\mathrm{Hz}$ or $1-\mathrm{Hz}$ Group.

\section{Affect questionnaires.}

Affect questionnaire. $10-\mathrm{Hz}$ group: No significant differences were found for the individual affect ratings. For mean affect ratings (ie the mean of all six ratings), ANOVA revealed a significant hemisphere by testing session interaction $(\mathrm{F}(1,3)=27.27, p<0.05)$, with subjects reporting lower affect following rTMS applied over the left hemisphere than following rTMS applied over the right hemisphere. 1-Hz Group: For both individual and mean affect ratings, ANOVA revealed no significant main effects or interactions.

Positive and negative affect scale. $10-\mathrm{Hz}$ group: For both mean positive and negative affect ratings, ANOVA revealed no significant main effects or interactions. $1-\mathrm{Hz}$ Group: For mean positive affect ratings, ANOVA revealed a significant main effect of hemisphere, with subjects rating themselves as having more positive affect during the rTMS session when the left hemisphere was stimulated $(\mathrm{F}(1,3)=9.69, p<0.05)$. Similarly, for mean negative affect ratings, ANOVA also revealed a significant main effect of hemisphere, with subjects rating themselves as having more negative affect during the rTMS session when the left hemisphere was stimulated $(\mathrm{F}(1,3)=24.61, p<0.05)$. As there was no interaction with session, it is possible that the higher positive and negative affect ratings reported during the left hemisphere session reflect a response bias for rating affect more liberally on that particular testing day.

Vitality scale. $10-\mathrm{Hz}$ group: For mean vitality ratings, ANOVA revealed a significant hemisphere by testing session interaction $(\mathrm{F}(1,3)=16.38, p<0.05)$, with subjects reporting lower vitality following rTMS applied over the left hemisphere than following rTMS applied over the right hemisphere. 1-Hz Group: For mean vitality ratings, ANOVA revealed a significant main effect of testing session, with subjects reporting a decrease in vitality after rTMS $(\mathrm{F}(1,3)=27.75, p<0.05)$.

Speech parameters. All means and standard deviations (adjusted for order of hemisphere stimulation) are presented in Table 1.

Mean range of $F_{0} .10-\mathrm{Hz}$ group: For mean range of $F_{0}$, ANOVA revealed a significant hemisphere by testing session interaction $(\mathrm{F}(1,3)=9.03, p=0.057)$, with subjects 
Table I Affect and Speech Measures

\begin{tabular}{|c|c|c|c|c|c|}
\hline & \multicolumn{3}{|c|}{ Left MDLFC } & \multicolumn{2}{|c|}{ Right MDLFC } \\
\hline & Group & Pre-rTMS & Post-rTMS & Pre-rTMS & Post-rTMS \\
\hline \multicolumn{6}{|l|}{ (a) Affect } \\
\hline \multirow[t]{4}{*}{ Affect questionnaire } & $10 \mathrm{~Hz}^{*}$ & 8.6 & 5 & 8.4 & 6.6 \\
\hline & & 2.5 & 3.46 & 2.4 & 3.24 \\
\hline & $\mathrm{I} \mathrm{Hz}$ & 7.8 & 8.2 & 8.6 & 7.8 \\
\hline & & 2.10 & 1.88 & 2.40 & 2.28 \\
\hline \multirow[t]{4}{*}{ Positive affect (PANAS) } & $10 \mathrm{~Hz}$ & 25.4 & 20.4 & 26.8 & 23.8 \\
\hline & & 0.51 & 2.13 & 2.08 & 1.77 \\
\hline & $\mathrm{I} \mathrm{Hz}$ & 26 & 22.6 & 27 & 20.8 \\
\hline & & 3.14 & 4.00 & 2.07 & 1.84 \\
\hline \multirow[t]{4}{*}{ Negative affect (PANAS) } & $10 \mathrm{~Hz}$ & 14.8 & 13.4 & 14.8 & 12.8 \\
\hline & & 1.39 & 0.60 & 1.46 & 0.37 \\
\hline & $\mathrm{I} \mathrm{Hz}$ & 16.8 & 14.4 & 15.8 & 14.2 \\
\hline & & 0.49 & 1.16 & 0.80 & 1.02 \\
\hline \multirow[t]{4}{*}{ Vitality scale } & $10 \mathrm{~Hz}^{*}$ & 4.46 & 3.63 & 4.8 & 4.51 \\
\hline & & 0.40 & 0.60 & 0.57 & 0.61 \\
\hline & $\mathrm{I} \mathrm{Hz}$ & 4.11 & 3.29 & 4.23 & 3.66 \\
\hline & & 0.40 & 0.44 & 0.23 & 0.40 \\
\hline \multicolumn{6}{|l|}{ (b) Speech } \\
\hline \multirow[t]{4}{*}{ Range of amplitude } & $10 \mathrm{~Hz}$ & 1220.47 & 935.07 & | 105.18 & 989.91 \\
\hline & & 278.50 & 186.83 & 220.79 & 258.10 \\
\hline & $\mathrm{I} \mathrm{Hz}$ & 934.05 & 842.29 & 998.63 & 944.15 \\
\hline & & 194.89 & 111.87 & 109.67 & 107.05 \\
\hline \multirow[t]{4}{*}{ Root-mean-square amplitude } & $10 \mathrm{~Hz}$ & 65.89 & 49.63 & 57.56 & 51.4 \\
\hline & & 19.38 & 11.19 & 14.63 & 14.79 \\
\hline & $\mathrm{I} \mathrm{Hz}$ & 50.47 & 44.37 & 51.96 & 48.36 \\
\hline & & 8.23 & 4.83 & 5.7 & 3.9 \\
\hline \multirow[t]{4}{*}{ Mean fundamental frequency } & $10 \mathrm{~Hz}$ & 250.26 & 247.71 & 251.19 & 243.38 \\
\hline & & 10.30 & 9.16 & 8.30 & 9.57 \\
\hline & $\mathrm{I} \mathrm{Hz}$ & 252.65 & 247.64 & 257.56 & 253.91 \\
\hline & & 8.57 & 6.51 & 5.29 & 8.64 \\
\hline \multirow{4}{*}{$\begin{array}{l}\text { Standard deviation of fundamental } \\
\text { frequency }\end{array}$} & $10 \mathrm{~Hz}$ & 47.86 & 45.83 & 51.22 & 49.07 \\
\hline & & 3.53 & 5.87 & 4.05 & 6.34 \\
\hline & $\mathrm{I} \mathrm{Hz}$ & 46.7 & 49.46 & 48.84 & 46.08 \\
\hline & & 1.24 & 3.01 & 2.53 & 2.08 \\
\hline \multirow[t]{4}{*}{ Range of fundamental frequency } & $10 \mathrm{~Hz}^{*}$ & 224.04 & 210.27 & 230.45 & 221.17 \\
\hline & & 17.62 & 17.87 & 16.32 & 18.01 \\
\hline & $\mathrm{I} \mathrm{Hz}$ & 222.93 & 227.02 & 224.09 & 219.33 \\
\hline & & 4.06 & 9.85 & 10.41 & 10.67 \\
\hline
\end{tabular}




\begin{tabular}{|c|c|c|c|c|c|}
\hline & \multicolumn{3}{|c|}{ Left MDLFC } & \multicolumn{2}{|c|}{ Right MDLFC } \\
\hline & Group & Pre-rTMS & Post-rTMS & Pre-rTMS & Post-rTMS \\
\hline \multicolumn{6}{|c|}{ (c) Working memory } \\
\hline \multirow{2}{*}{ Response time } & $10 \mathrm{~Hz}$ & 1062.48 & 993.66 & 1046.82 & 999.99 \\
\hline & & 93.57 & 85.55 & 133.44 & 113.50 \\
\hline \multirow[t]{4}{*}{ Percent correct } & $10 \mathrm{~Hz}$ & 90 & 96.67 & 95 & 94.17 \\
\hline & & 7.04 & 1.56 & 5 & 3.86 \\
\hline & $\mathrm{I} \mathrm{Hz}$ & 98.33 & 96.67 & 95.83 & 97.5 \\
\hline & & 1.02 & 1.56 & 1.86 & 1.66 \\
\hline
\end{tabular}

* Indicates a significant interaction $(p<0.05)$, the standard error of the mean is indicated in italics.

displaying lower range of $F_{0}$ following rTMS applied over the left hemisphere than following rTMS applied over the right hemisphere. 1-Hz Group: For mean range of $F_{0}$, ANOVA revealed no significant main effects or interactions.

Rms amplitude, range of amplitude, mean $F_{0}$, and standard deviation of $F_{0}$ : For each of these parameters and for both the $10-$ and $1-\mathrm{Hz}$ Group, ANOVA revealed no significant main effects or interactions.

\section{Working memory.}

10-Hz Group and 1-Hz Group: For both mean response time and accuracy, ANOVA revealed no significant main effects or interactions.

\section{Experiment 2: rTMS Effects on Cerebral Blood Flow}

During debriefing, one subject revealed that she was sleep deprived (only $2 \mathrm{~h}$ sleep) upon arrival at one of the TMSPET sessions (10-Hz Conditioning); her data from both sessions were excluded from further analyses.

Affect questionnaire. For individual affect ratings and the mean of the six ratings, ANOVA with rTMS session $(10-\mathrm{Hz}$ Conditioning, $1-\mathrm{Hz}$ Conditioning) and testing point (preTMS-PET, post-TMS-PET) as within-subject factors and the order in which the conditioning rTMS was applied $(10-\mathrm{Hz}$ or $1-\mathrm{Hz}$ Conditioning during session 1) as a covariate revealed no significant main effects or interactions. It is of note that during debriefing with the experimenter, there were no systematic reports of subjective differences between $10-\mathrm{Hz}$ Conditioning or $1-\mathrm{Hz}$ Conditioning sessions. The lack of significant affect differences were not surprising, given the unavoidable confounds of the TMS-PET session (eg frequent interaction with PET technicians, IV catheter, bite-bar).

Seed-voxel PLS. For the $10-\mathrm{Hz}$ Conditioning session, a significant increase in CBF was observed in the left MDLFC at the site of stimulation $(x=-38, y=21, z=37, t=3.82)$ when subtracting the average of the Probes 2,3 , and 4 (ie the scans immediately following the between-scan stimulation) from Probe 5. Importantly, as noted in Figure 2b, the location of the observed CBF increase overlaps with the location of the average TMS coil placement. This finding of a significant increase in CBF during the last probe scan acquired $10 \mathrm{~min}$ after the last conditioning series of $10-\mathrm{Hz}$ rTMS is consistent with our previous study (Paus et al, 2001). Irrespective of the subtraction used, a significant change in $\mathrm{CBF}$ at the site of stimulation was found only for the $10-\mathrm{Hz}$ Conditioning session. As a result, seed-voxel PLS analysis was not performed for the $1-\mathrm{Hz}$ Conditioning session.

For the $10-\mathrm{Hz}$ Conditioning session, the seed-voxel PLS analysis for the left MDLFC yielded two significant latent variables, LV1 $(p<0.05)$ and $\operatorname{LV} 2(p=0.08)$. LV1 identified a common pattern of brain regions that accounted for $47 \%$ of the total covariance (see Figure $3 \mathrm{a}$ ). The scan profile of LV1, shown in Figure $3 \mathrm{~b}$, indicated that the pattern of brain areas identified by this variable was moderately correlated with the left MDLFC and that this correlation did not vary as a function of rTMS. Given that the profile for the baseline did not differ from the rTMS scans, it is likely that LV1 represents a network of brains regions associated with the MDLFC that are active at rest. The stereotaxic coordinates corresponding to the peak saliences (greater than 3) for these regions (positive saliences are represented in yellow and negative saliences in blue) are listed in Table $2 \mathrm{a}$.

LV2 accounted for $23 \%$ of the total covariance. The pattern of brain regions demonstrating a relationship with the MDLFC that were identified by this variable distinguished between the scans and changed as a function of rTMS (Figure 4a). The stereotaxic coordinates for positive and negative saliences greater than 3 are listed in Table $2 \mathrm{~b}$. Figure $4 \mathrm{~b}$ shows that activity in the regions labeled in yellow became positively correlated with that in MDLFC towards the end of the rTMS session, that is Probes 4 and 5. Thus, the increment in MDLFC activity induced by rTMS during Probe 5 (see univariate results above) was followed by an increment in activity in the perigenual anterior cingulate 


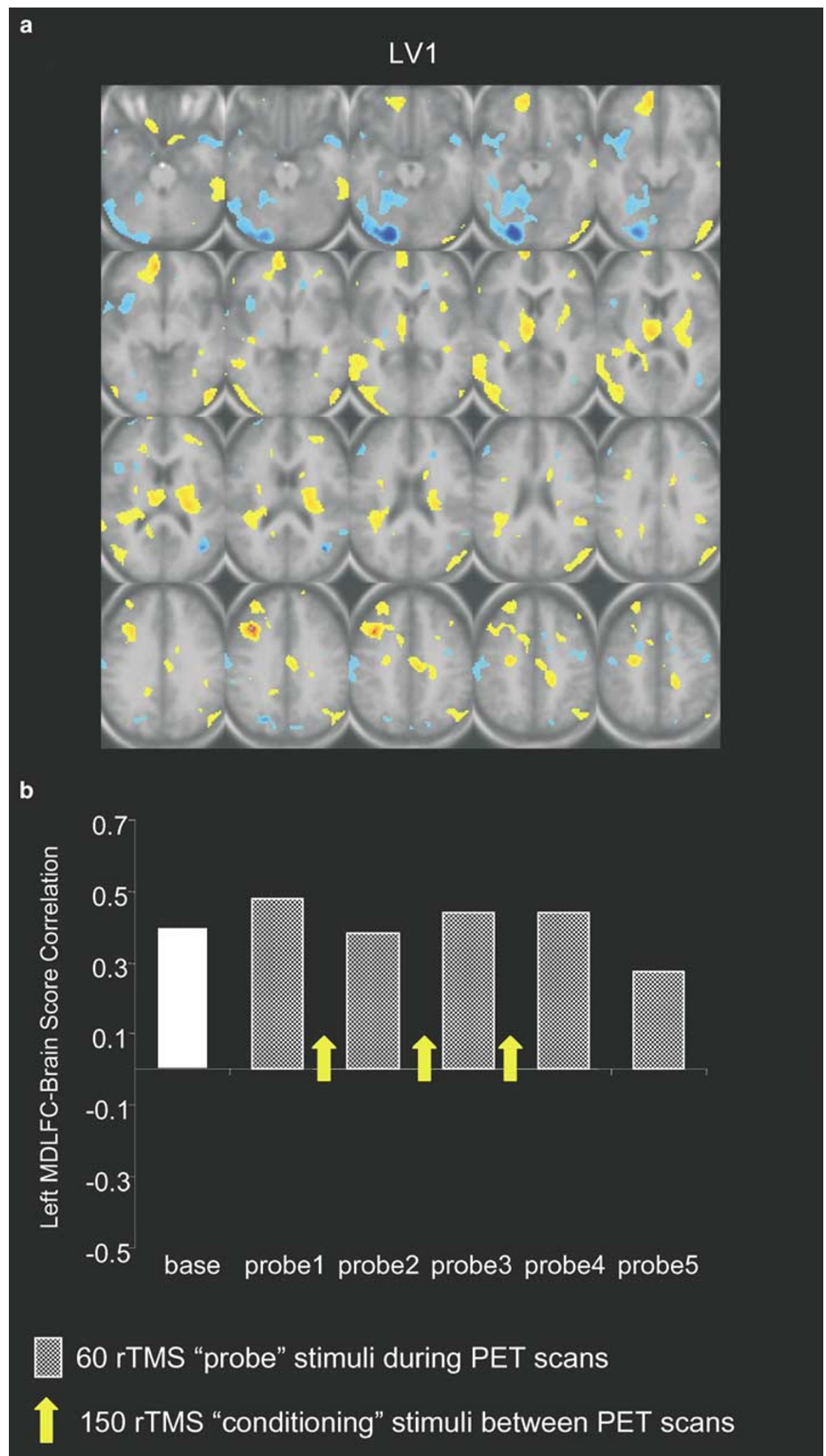

Figure 3 The singular image and scan profile of LVI. Shown above, the singular image (a) and scan profile (b) for LVI. The singular image contains a weighted linear combination of brain regions that, as a whole, covaried with the left MDLFC. All positive (yellow) and negative (blue) saliences for LVI are located in Table 2a. As indicated in (b), the scan profile revealed that the pattern of brain areas identified in LVI was moderately correlated with the left MDLFC and that this correlation did not vary as a function of rTMS. 


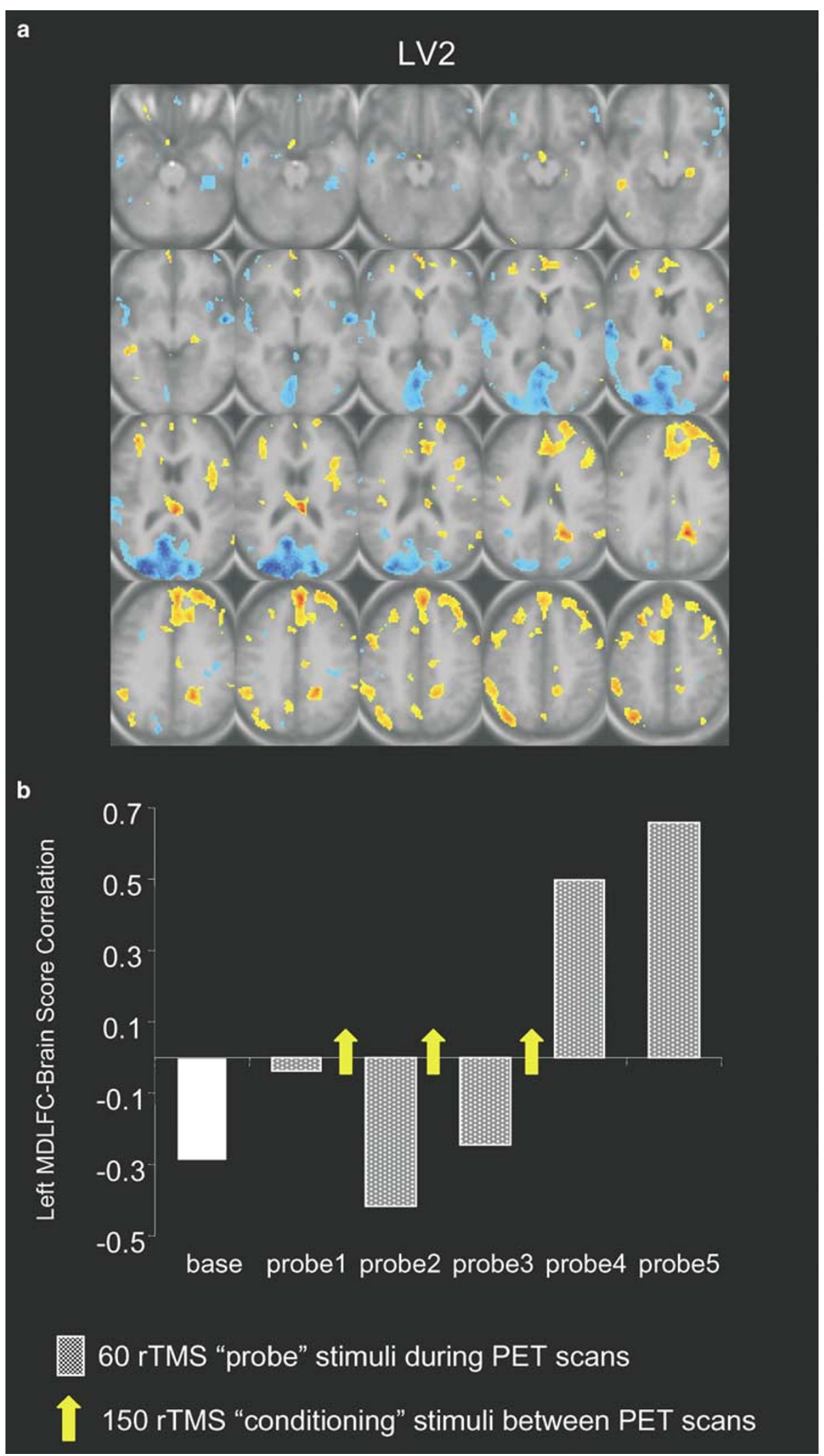

Figure 4 The singular image and scan profile of LV2. Shown above, the singular image (a) and scan profile (b) for LV2. The singular image contains a weighted linear combination of brain regions that, as a whole, covaried with the left MDLFC. All positive (yellow) and negative (blue) saliences for LV2 are located in Table 2b. As indicated in (b), activity in the regions labeled in yellow became positively correlated with that in MDLFC towards the end of the rTMS session, that is, during Probes 4 and 5; the regions in blue became strongly negatively correlated. 
Table 2 Positive and Negative Saliences of LVI and LV2

\begin{tabular}{|c|c|c|c|c|c|}
\hline & Hemisphere & $x$ & $y$ & $\mathbf{z}$ & Salience \\
\hline \multicolumn{6}{|l|}{ (a) LV I } \\
\hline \multicolumn{6}{|l|}{ Positive saliences } \\
\hline Middle frontal gyrus (seed) & $L$ & -38 & 21 & 37 & \\
\hline \multirow[t]{3}{*}{ Middle frontal gyrus } & $L$ & -38 & 24 & 36 & 23.0941 \\
\hline & $\mathrm{R}$ & 46 & 34 & 44 & 3.798 \\
\hline & $\mathrm{R}$ & 28 & 10 & 44 & 3.3764 \\
\hline Gyrus rectus & $\mathrm{R}$ & -8 & 52 & -8 & II.0462 \\
\hline \multirow[t]{2}{*}{ Thalamus } & $L$ & -6 & -18 & 8 & 9.0644 \\
\hline & $\mathrm{R}$ & 26 & -24 & 12 & 6.894 \\
\hline \multirow[t]{2}{*}{ Precentral gyrus } & $L$ & -26 & -14 & 48 & 6.894 \\
\hline & $\mathrm{R}$ & 52 & -6 & 60 & 3.889 \\
\hline \multirow[t]{2}{*}{ Inferior occipital gyrus } & $L$ & -40 & -92 & 0 & 6.3541 \\
\hline & $\mathrm{R}$ & 50 & -82 & -4 & 6.1849 \\
\hline Middle occipital gyrus & $\mathrm{R}$ & 52 & -76 & 32 & 4.9169 \\
\hline Caudate nucleus & $\mathrm{R}$ & 16 & 6 & 24 & 4.7399 \\
\hline Inferior frontal gyrus & $\mathrm{R}$ & 40 & 46 & 4 & 4.6643 \\
\hline Anterior cingulate gyrus & $L$ & -20 & 36 & 12 & 3.5803 \\
\hline Fusiform gyrus & $\mathrm{R}$ & 34 & -58 & -8 & 3.002 \\
\hline \multicolumn{6}{|l|}{ Negative saliences } \\
\hline \multirow[t]{2}{*}{ Fusiform gyrus } & $L$ & -22 & -90 & -20 & 10.8903 \\
\hline & & -44 & -34 & -16 & 3.6448 \\
\hline Inferior parietal lobule & $\mathrm{R}$ & 40 & -70 & 16 & 7.9152 \\
\hline \multirow[t]{2}{*}{ Precuneus } & $L$ & -22 & -74 & 36 & 6.6209 \\
\hline & $\mathrm{R}$ & 10 & -58 & 60 & 3.7902 \\
\hline \multirow[t]{2}{*}{ Superior temporal gyrus } & $\mathrm{R}$ & 40 & 12 & -28 & 5.8543 \\
\hline & & 60 & -52 & 16 & 3.6932 \\
\hline \multirow[t]{3}{*}{ Middle frontal gyrus } & $L$ & -52 & 36 & 24 & 5.4135 \\
\hline & $L$ & -36 & 0 & 52 & 5.2362 \\
\hline & $\mathrm{R}$ & 34 & 4 & 28 & 3.0069 \\
\hline Insula & $L$ & -42 & 10 & -8 & 5.133 \\
\hline Postcentral gyrus & $L$ & -40 & -34 & 56 & $5.099 \mid$ \\
\hline Supplementary motor area & $\mathrm{R}$ & 16 & -4 & 52 & 4.2175 \\
\hline Lentiform nucleus & $L$ & -28 & -20 & 0 & 4.0461 \\
\hline Superior frontal gyrus & $L$ & -30 & 64 & 16 & 3.9408 \\
\hline Claustrum & $L$ & -28 & 18 & 12 & 3.8774 \\
\hline Anterior cingulate gyrus & $\mathrm{R}$ & 16 & 34 & -4 & 3.8227 \\
\hline Inferior temporal gyrus & $L$ & -60 & -2 & -28 & 3.379 \\
\hline
\end{tabular}

\section{(b) LV2}

Positive saliences

Middle frontal gyrus (seed)

Thalamus

Superior frontal gyrus

Middle temporal gyrus

Middle frontal gyrus

Postcentral gyrus

$\begin{array}{lr}L & -38 \\ R & 8 \\ L & -28 \\ R & 62 \\ L & -48 \\ L & -36 \\ & -52 \\ L & -28\end{array}$

21
-32
24
-68
-38
42
6
-40


Table 2 (Continued)

\begin{tabular}{|c|c|c|c|c|c|}
\hline & Hemisphere & $x$ & $y$ & $\mathbf{z}$ & Salience \\
\hline Parahippocampal gyrus & $\mathrm{R}$ & 24 & -26 & -12 & 4.1502 \\
\hline \multirow[t]{2}{*}{ Caudate nucleus } & $\mathrm{L}$ & -12 & 24 & 8 & 4.125 \\
\hline & $\mathrm{R}$ & 14 & -6 & 24 & 3.024 \\
\hline Posterior cingulate gyrus & $\mathrm{R}$ & 4 & -14 & 32 & 4.033 \\
\hline Precuneus & & 0 & -74 & 44 & 3.7466 \\
\hline Insula & $\mathrm{L}$ & -38 & -24 & 20 & 3.1863 \\
\hline Anterior cingulate gyrus & $\mathrm{L}$ & -2 & 24 & 0 & 3.1254 \\
\hline \multicolumn{6}{|l|}{ Negative saliences } \\
\hline Middle occipital gyrus & L & -26 & -90 & 16 & 7.4345 \\
\hline \multirow[t]{2}{*}{ Inferior frontal gyrus } & $\mathrm{R}$ & 58 & 36 & -12 & 5.2161 \\
\hline & $\mathrm{L}$ & -30 & 30 & -16 & 3.9894 \\
\hline Inferior temporal gyrus & L & -50 & -12 & -20 & 5.1435 \\
\hline \multirow[t]{2}{*}{ Superior parietal lobule } & $\mathrm{L}$ & -24 & -64 & 52 & 5.093 \\
\hline & $\mathrm{R}$ & 20 & -46 & 60 & 3.6217 \\
\hline Precuneus & $\mathrm{R}$ & 10 & -58 & 64 & 4.791 \\
\hline Insula & $\mathrm{R}$ & 36 & -20 & 8 & 4.0506 \\
\hline Inferior parietal lobule & L & -38 & -54 & 24 & 3.963 \\
\hline Precentral gyrus & L & -26 & -18 & 64 & 3.8858 \\
\hline Middle frontal gyrus & $\mathrm{L}$ & -52 & 48 & -4 & 3.6193 \\
\hline
\end{tabular}

gyrus corresponding to cytoarchitectonic area 24a (as defined by Vogt et al, 1995; see Table $2 \mathrm{~b}$ and Figure 4a), as well as in the insula, thalamus, parahippocampal gyrus, and caudate nucleus. In contrast, increments in MDLFC activity were associated with reductions in activity in areas depicted in blue including the amygdala, middle frontal gyrus (Brodmann area 9), inferior frontal gyrus, gyrus rectus, and the inferior and superior parietal lobules.

\section{DISCUSSION}

The goal of the current study was to investigate the neural substrates of rTMS-induced changes in affective state. In Experiment 1, we combined $10-\mathrm{Hz}$ rTMS applied over the left MDLFC with a speech task to examine rTMS-induced changes in paralinguistic aspects of speech production. In Experiment 2, we combined $10-\mathrm{Hz}$ rTMS applied over the left MDLFC with PET to examine rTMS-induced changes in the functional connectivity of the MDLFC. Taken together, the results of the two experiments revealed that $10-\mathrm{Hz}$ rTMS applied over the left MDLFC decreased affect and pitch variation in speech and increased the functional connectivity between the site of stimulation and other brain areas associated with affect, such as the ACC. No robust changes in behavior or brain activity were observed following $1-\mathrm{Hz}$ rTMS.

\section{0-Hz rTMS Decreases Affect and Pitch Variation}

The results of Experiment 1 revealed that $10-\mathrm{Hz}$ rTMS applied over the left MDLFC resulted in lower affect, vitality, and pitch variation in speech. Our finding of significant hemisphere of stimulation (left MDLFC, right MDLFC) by testing point (pre-rTMS, post-rTMS) interactions for the affect and speech measures supports a special role for the left MDLFC in the neural pathways regulating affect and rules out the possibility that the changes in affect observed following left MDLFC stimulation could have been due to the 'knocking' of the TMS coil during high-frequency TMS or lying quietly with eyes closed for the 30-min rTMS session. While in the 1-Hz Group rTMS applied over the left and right MDLFC decreased self-reported vitality, we observed no interaction between site of stimulation and testing session and thus cannot be certain if the observed decreases in vitality are related to rTMS-induced changes in brain activity or other aspects of the experimental procedure, as described above. 
In general, the affect changes observed in Experiment 1 are consistent with previous work demonstrating that $10-\mathrm{Hz}$ rTMS applied over the left MDLFC in healthy volunteers decreased self-reported affect (ie lower positive affect or higher negative affect). Importantly, using a novel TMSspeech paradigm our results also demonstrate the utility of examining rTMS-induced changes in specific aspects of affective symptomatology, in this case, pitch variation in speech. There are two major strengths of this paradigm: (1) consistent with our previous behavioural study (Barrett and Paus, 2002), we were able to obtain an objective index of affective state and (2) we were able to demonstrate rTMSinduced changes in a behavior linked to the ACC (Barrett et al, 2004).

\section{0-Hz rTMS Increases the Connectivity of the Left MDLFC}

The results of Experiment 2 demonstrated that $10-\mathrm{Hz}$ rTMS applied over the MDLFC influenced $\mathrm{rCBF}$ in a network of brain regions implicated in affect, many of which are presumed to be directly connected with the stimulated cortex. Specifically, seed-voxel PLS analyses revealed a pattern of brain regions where CBF became positively correlated with CBF in the left MDLFC during Probes 4 and 5 ; this result is consistent with the univariate statistical analysis showing a significant increment of CBF for MDLFC during Probe 5. These regions included the perigenual anterior cingulate gyrus, insula, thalamus, parahippocampal gyrus, and the caudate nucleus. Brain regions following the opposite pattern (ie moderate positive covariation becoming strong negative covariation) included the middle frontal gyrus, gyrus rectus, amygdala, and inferior and superior parietal lobules. Our finding of increased positive MDLFCACC covariation following the last series of Conditioning $10-\mathrm{Hz}$ rTMS is also consistent with the CBF increases in the ACC observed in our previous TMS-PET study (Paus et al, 2001).

\section{Connectivity of the MDLFC and the Pathophysiology of Depression}

Many of the brain regions found to be influenced by rTMS and identified as being functionally connected to the MDLFC have also been implicated in the pathophysiology of depression (for reviews, see Drevets, 2001; Davidson et al, 2002; Mayberg, 2003). For example, in a double-blind placebo-controlled study of depressed patients, clinical improvement following six weeks of fluoxetine was associated with metabolic decreases in the caudate nucleus, hippocampus, and insula and metabolic increases in the parietal and prefrontal cortices (Mayberg et al, 2000). Similar patterns of increases and decreases in brain metabolism have also been observed following fluoxetine treatment of depression in patients with Parkinson's disease (reviewed in Mayberg, 2003). In our study, however, and consistent with the increases in sadness that have been observed following 10-Hz rTMS, rTMS-induced increases in MDLFC blood flow were associated with greater activity in the hippocampus and the caudate nucleus and less activity in prefrontal cortices and parietal cortices. While the similarities between past antidepressant studies and our current work are encouraging, it is of note that there is a great deal of interstudy variability in the pattern of brain regions displaying blood flow and metabolic changes in depressed patients, both at pre- and post-treatment. This is thought to be related to the heterogeneity of patient groups in terms of symptom expression, a phenomenon possibly mediated by the existence of discrete but functionally interconnected neural pathways underlying specific aspects of depressive symptomatology (Drevets, 2001; Mayberg, 2003). Despite the known interstudy differences, the brain regions most commonly implicated in depression and consistent with the results of our current study include the MDLFC, orbitofrontal cortex, thalamus, hippocampus and importantly, the ACC (Drevets, 2001; Mayberg, 2003).

\section{Increased MDLFC-ACC Connectivity and Pitch Variation in Speech}

In our study, $\mathrm{CBF}$ in a perigenual region of the ACC became strongly positively correlated with the left MDLFC during Probes 4 and 5; this increase in functional connectivity was associated with increased blood flow in the MDLFC. Increases in neural activity in similar regions of the ACC have been observed during depression (Kennedy et al, 2001; Videbech et al, 2002) and experimentally induced sadness (eg George et al, 1995; Liotti et al, 2000), and baseline activity in this region has been shown to predict response to antidepressant treatment (Mayberg et al, 1997, Brannan et al, 2000; Pizzagalli et al, 2001). In addition, higher glucose metabolism and perfusion in perigenual aspects of the ACC have been found to be positively correlated with both severity of depression and psychomotor retardation (Ebmeier et al, 1997; Dunn et al, 2002). With regard to the role of the ACC in depression and affect, we have proposed that the ACC acts as an interface between action and emotion (Paus, 2001). The anatomical corticocortical connectivity between the ACC and both the prefrontal and motor cortices provides pathways for communication between the cognitive and motor systems (Barbas and Pandya, 1989; Dum and Strick, 1991; Morecraft and Van Hoesen, 1992, 1993; Bates and Goldman-Rakic, 1993; Picard and Strick, 1996). Emotional and motivational states can further influence and/or involve the ACC via regulatory input from brainstem monoaminergic nuclei as well as via input from the amygdala, thalamus, and ventral striatum (Barbas and De Olmos, 1990; Barbas et al, 1991; Kunishio and Haber, 1994; Ongur and Price, 2000). In light of the proposed role of the ACC in paralinguistic aspects of speech during changes in emotion and motivation, it is important to note that the regions of ACC surrounding the genu of the corpus callosum have numerous connections with cortical and subcortical regions involved in vocalization, including those in the periaqueductal gray (Jurgens, 1976a, b, 1983; Muller-Preuss and Jurgens, 1976; Morecraft and Van Hoesen, 1993; An et al, 1998).

There is ample evidence for a direct role of the ACC in regulating aspects of speech during emotional states. In monkeys, damage to the subcallosal, perigenual, and supracallosal ACC has been shown to prevent the volitional initiation of vocal responses in emotionally valenced situations (Sutton, Larson and Lindeman, 1974; Aitken, 1981; MacLean and Newman, 1988). Further, in our 
previous study in humans combining a mood inductionspeech task procedure (Barrett and Paus, 2002) with functional magnetic resonance imaging, the blood oxygen level-dependent signal in the ACC was found to be the only significant 'neural' predictor of pitch variation during sad affect, with increased BOLD signal in the ACC associated with both decreased pitch variation and more negative affect (Barrett, Pike and Paus, 2004). Thus, in light of our brain imaging findings and the posited role of the ACC in speech, we may propose two potential mechanisms for the $10-\mathrm{Hz}$ rTMS-induced changes in pitch variation observed in our behavioral study. First, the observed decreases in pitch variation could be a direct result of rTMS-induced increases in neural activity in the ACC. In this model, rTMS applied over the left MDLFC influences neural activity in a number of cortical and subcortical pathways that underlie specific aspects of affective symptomatology, including a frontocingulate circuit. A second potential mechanism for rTMSinduced changes in pitch variation is that $10-\mathrm{Hz}$ rTMS applied over the MDLFC may influence neural activity in the prefrontal cortex and/or other affect-relevant brain circuits (eg frontothalamic, frontostriatal), which influences affective state, which in turn mediates pitch variation possibly through the recruitment of the ACC. As we are unable to stimulate the ACC directly and can only infer the modulation of neural activity in the ACC via MDLFC stimulation, this second model cannot be ruled out.

\section{The Effect of 1-Hz rTMS on Brain Activity and Behaviour}

In Experiment 1, 1-Hz rTMS applied over the left and right MDLFC resulted in no significant changes in affect or speech. These negative findings may be surprising given the reports of the therapeutic effect of $1-\mathrm{Hz}$ rTMS applied over the right MDLFC in depression (eg Klein et al, 1999). Compared to that of $10-\mathrm{Hz}$ rTMS, however, the mood effects of 1-Hz rTMS have been far less frequently reported (see the Avery-George-Holtzheimer Database of rTMS Depression studies, available at: http://www.ists.unibe.ch/TMSAvery. $\mathrm{htm})$. In addition, in the $1-\mathrm{Hz}$ Conditioning session of Experiment 2, we did not observe any significant changes in blood flow at the site of stimulation, a finding that is inconsistent with the results of previous studies in healthy volunteers investigating the effect of $1-\mathrm{Hz}$ rTMS applied over the MDLFC on neural activity (eg Nahas et al, 2001; Speer et al, 2003). It should be emphasized that, in contrast to these previous investigations where $1-\mathrm{Hz}$ rTMS was applied during brain imaging, in our $1-\mathrm{Hz}$ Conditioning session $1-\mathrm{Hz}$ rTMS was applied between the scans. This makes direct comparison between previous findings and that of the current investigation difficult. Further, the presumably more robust $10-\mathrm{Hz}$ rTMS applied during the probe scans might have diminished the effect of the $1-\mathrm{Hz}$ conditioning rTMS applied between the probe scans. Thus, there are at least two possibilities for the observed inconsistencies in the current results of Experiments and 1 and 2 and that of past research: (1) 1-Hz rTMS does not have a robust effect on blood flow and behavior and/or (2) the effect of $1-\mathrm{Hz}$ rTMS on blood flow and behavior is sensitive to differences in stimulation parameters (eg number of TMS stimuli, intensity of stimulation) and the stimulation paradigm (eg between-scan stimulation $v s$ no between-scan stimulation). Nonetheless, despite the consistent negative findings for $1-\mathrm{Hz}$ rTMS observed in Experiments 1 and 2, it would be speculative to draw any conclusions about the effect of $1-\mathrm{Hz}$ rTMS on mood and brain activity at this point.

\section{Limitations}

There are a number of limitations to both Experiment 1 and Experiment 2 that must be addressed. First, as we did not image the brain during our speech task we can only speculate that the $10-\mathrm{Hz}$ rTMS-induced changes in pitch variation observed in Experiment 1 may be related to the $10-\mathrm{Hz}$ rTMS-induced changes in neural activity observed in Experiment 2. Thus, we cannot be sure if the increases in MDLFC-ACC connectivity and ACC blood flow observed in Experiment 2 are dependent on either (1) the additional rTMS stimuli (ie 450 rTMS stimuli applied in Experiment 1 vs 750 rTMS stimuli applied in Experiment 2) and/or (2) the paradigm of high-frequency conditioning rTMS followed by low-frequency probe rTMS. Although it represents a limitation in our ability to link the findings of our two experiments, our strategy of conducting two separate studies was thought to be the least confounded approach to assess rTMS effects on affect and speech and MDLFC connectivity.

Second, the mood and speech changes observed in Experiment 1 following $10-\mathrm{Hz}$ rTMS applied over the left MDLFC are relatively small. The magnitude of behavioral change is comparable, however, to that previously observed in healthy volunteers following a brief mood induction (Barrett and Paus, 2002; Barrett et al, 2004). Further, it is possible that larger effects on mood and speech could be induced by repeating the stimulation over a number of sessions, as in rTMS treatment protocols conducted in depressed patients.

\section{Conclusion}

Our results suggest that following $10-\mathrm{Hz}$ rTMS applied over the left MDLFC, changes in affect and affect-relevant behavior may be related to changes in neural activity in a functionally connected network of brain regions widely implicated in affective states, including a frontocingulate circuit. While the implications of our rTMS results in healthy subjects for the antidepressant effect of rTMS are uncertain, especially given the complexity of the depressive syndrome relative to brief changes in mood states as well as the opposite rTMS-induced mood effects observed in depressed patients compared to healthy subjects, we feel that our investigation of rTMS 'acute' effects in affectrelevant brain regions provides useful information about potential rTMS mechanisms of action in the context of mood. Further, our results may also bring us a step closer to understanding the neural pathways underlying the expression of specific aspects of affective symptomatology. Through future work, our knowledge of rTMS-induced mood effects and the pathophysiology of affective states may be enhanced by employing similar behavioral and brain imaging paradigms to investigate rTMS 'acute' effects 
in depressed patients as well as in healthy subjects following mood induction.

\section{ACKNOWLEDGEMENTS}

This research was supported in part by a Natural Sciences and Engineering Research Council of Canada (NSERC) post-graduate scholarship awarded to the first author and an operating grant from the Canadian Institutes for Health Research (CIHR) to Tomáš Paus.

\section{REFERENCES}

Aitken PG (1981). Cortical control of conditioned and spontaneous vocal behaviour in rhesus monkeys. Brain Lang 13: 171-184.

An X, Bandler R, Ongur D, Price JL (1998). Prefrontal cortical projections to longitudinal columns in the midbrain periaqueductal gray in macaque monkeys. J Comp Neurol 401: 455-479.

Barbas H (2000). Complementary roles of prefrontal cortical regions in cognition, memory, and emotion in primates. $A d v$ Neurol 84: 87-110.

Barbas H, De Olmos J (1990). Projections from the amygdala to basoventral and mediodorsal prefrontal regions in the rhesus monkey. J Comp Neurol 300: 549-571.

Barbas H, Henion TH, Dermon CR (1991). Diverse thalamic projections to the prefrontal cortex in the rhesus monkey. J Comp Neurol 313: 65-94.

Barbas H, Pandya DN (1989). Architecture and intrinsic connections of the prefrontal cortex in the rhesus monkey. J Comp Neurol 286: 353-375.

Barrett J, Paus T (2002). Affect-induced changes in speech production. Exp Brain Res 146: 531-537.

Barrett J, Pike GB, Paus T (2004). The role of the anterior cingulate cortex in pitch variation during sad affect. Eur J Neurosci 19: 458-464.

Bates JF, Goldman-Rakic PS (1993). Prefrontal connections of medial motor areas in the rhesus monkey. J Comp Neurol 336: 211-228.

Brannan SK, Mayberg HS, McGinnis S, Silva JA, Tekell J, Mahurin $\mathrm{RK}$ et al (2000). Cingulate metabolism predicts treatment response: a replication. Biol Psychiatry 47: 107S.

Bush G, Luu P, Posner MI (2000). Cognitive and emotional influences in anterior cingulate cortex. Trends Cogn Sci 4: 215-222.

Catafau AM, Perez V, Gironell A, Martin JC, Kulisevsky J, Estorch $M$ et al (2001). SPECT mapping of cerebral activity changes induced by repetitive transcranial magnetic stimulation in depressed patients. A pilot study. Psychiatry Res 106: $151-160$

Chen R (2000). Studies of human motor physiology with transcranial magnetic stimulation. Muscle Nerve Suppl 9: S26-S32.

Collins DL, Neelin P, Peters TM, Evans AC (1994). Automatic 3D intersubject registration of MR volumetric data in standardized Talairach space 33. J Comput Assist Tomogr 18: 192-205.

Conca A, Peschina W, Konig P, Fritzsche H, Hausmann A (2002). Effect of chronic repetitive transcranial magnetic stimulation on regional cerebral blood flow and regional cerebral glucose uptake in drug treatment-resistant depressives. A brief report. Neuropsychobiology 45: 27-31.

Davidson RJ, Pizzagalli D, Nitschke JB, Putnam K (2002). Depression: perspectives from affective neuroscience. Annu Rev Psychol 53: 545-574.

Della-Maggiore V, Sekular AB, Grady CL, Bennett PJ, Sekular R, McIntosh AR (2000). Corticolimbic interactions associated with performance on a short-term memory task are modified by age. J Neurosci 20: 8410-8416.

Devinsky O, Morrell MJ, Vogt BA (1995). Contributions of anterior cingulate cortex to behaviour. Brain 118: 279-306.

Drevets WC (2000a). Neuroimaging studies of mood disorders. Biol Psychiatry 48: 813-829.

Drevets WC (2000b). Functional anatomical abnormalities in limbic and prefrontal cortical structures in major depression. Prog Brain Res 126: 413-431.

Drevets WC (2001). Neuroimaging and neuropathological studies of depression: implications for the cognitive-emotional features of mood disorders. Curr Opin Neurobiol 11: 240-249.

Dum RP, Strick PL (1991). The origin of corticospinal projections from the premotor areas in the frontal lobe. J Neurosci 11: 667-689.

Dunn RT, Kimbrell TA, Ketter TA, Frye MA, Willis MW, Luckenbaugh DA et al (2002). Principal components of the Beck Depression Inventory and regional cerebral metabolism in unipolar and bipolar depression. Biol Psychiatry 51: 387-399.

Ebmeier KP, Cavanagh JT, Moffoot AP, Glabus MF, O'Carroll RE, Goodwin GM (1997). Cerebral perfusion correlates of depressed mood. Br J Psychiatry 170: 77-81.

Efron B, Tibshirani R (1986). Bootstrap methods for standard errors, confidence intervals and other measures of statistical accuracy. Stat Sci 1: 54-77.

First MB, Spitzer RL, Gibbon M, Williams JBW (2002). Structured Clinical Interview for DSM-IV-TR Axis I Disorders, Research Version, Non-patient Edition. Biometrics Research, New York State Psychiatric Institute: New York.

George MS, Ketter TA, Parekh PI, Horwitz B, Herscovitch P, Post RM (1995). Brain activity during transient sadness and happiness in healthy women. Am J Psychiatry 152: 341-351.

George MS, Lisanby SH, Sackeim HA (1999). Transcranial magnetic stimulation: applications in neuropsychiatry. Arch Gen Psychiatry 56: 300-311.

George MS, Wassermann EM, Williams WA, Steppel J, PascualLeone A, Basser P et al (1996). Changes in mood and hormone levels after rapid-rate transcranial magnetic stimulation (rTMS) of the prefrontal cortex. J Neuropsychiatry Clin Neurosci 8: $172-180$.

Gershon AA, Dannon PN, Grunhaus L (2003). Transcranial magnetic stimulation in the treatment of depression. Am J Psychiatry 160: 835-845.

IEEE Standards Publication No. 297 (1969). IEEE recommended practice for speech quality measurements. IEEE Trans Audio Electroacoust 17.

Jurgens U (1976a). Reinforcing concomitants of electrically elicited vocalizations. Exp Brain Res 26: 203-214.

Jurgens U (1976b). Projections from the cortical larynx area in the squirrel monkey. Exp Brain Res 25: 401-411.

Jurgens U (1983). Afferent fibers to the cingular vocalization region in the squirrel monkey. Exp Neurol 80: 395-409.

Kennedy SH, Evans KR, Kruger S, Mayberg HS, Meyer JH, McCann S et al (2001). Changes in regional brain glucose metabolism measured with positron emission tomography after paroxetine treatment of major depression. Am J Psychiatry 158: 899-905.

Kimbrell TA, Little JT, Dunn RT, Frye MA, Greenberg BD, Wassermann EM et al (1999). Frequency dependence of antidepressant response to left prefrontal repetitive transcranial magnetic stimulation (rTMS) as a function of baseline cerebral glucose metabolism. Biol Psychiatry 46: 1603-1613.

Klein E, Kreinin I, Chistyakov A, Koren D, Mecz L, Marmur S et al (1999). Therapeutic efficacy of right prefrontal slow repetitive transcranial magnetic stimulation in major depression: a double-blind controlled study. Arch Gen Psychiatry 56: $315-320$ 
Kunishio K, Haber SN (1994). Primate cingulostriatal projection: limbic striatal versus sensorimotor striatal input. J Comp Neurol 350: 337-356.

Liotti M, Mayberg HS, Brannan SK, McGinnis S, Jerabek P, Fox PT (2000). Differential limbic-cortical correlates of sadness and anxiety in healthy subjects: implications for affective disorders. Biol Psychiatry 48: 30-42.

MacLean PD, Newman JD (1988). Role of midline frontolimbic cortex in production of the isolation call of squirrel monkeys. Brain Res 450: 111-123.

Martin JD, George MS, Greenberg BD, Wassermann EM, Schlaepfer TE, Murphy DL et al (1997). Mood effects of prefrontal repetitive high-frequency TMS in healthy volunteers. CNS Spectrums 2: 53-54.

Martin JL, Barbanoj MJ, Schlaepfer TE, Thompson E, Perez V, Kulisevsky J (2003). Repetitive transcranial magnetic stimulation for the treatment of depression. Systematic review and metaanalysis. $\mathrm{Br}$ J Psychiatry 182: 480-491.

Mayberg HS (2003). Modulating dysfunctional limbic-cortical circuits in depression: towards development of brain-based algorithms for diagnosis and optimised treatment. $\mathrm{Br}$ Med Bull 65: 193-207.

Mayberg HS, Brannan SK, Mahurin RK, Jerabek PA, Brickman JS, Tekell JL et al (1997). Cingulate function in depression: a potential predictor of treatment response. Neuroreport 8: 10571061.

Mayberg HS, Brannan SK, Tekell JL, Silva JA, Mahurin RK, McGinnis S et al (2000). Regional metabolic effects of fluoxetine in major depression: serial changes and relationship to clinical response. Biol Psychiatry 48: 830-843.

McIntosh AR, Bookstein FL, Haxby JV, Grady CL (1996). Spatial pattern analysis of functional brain images using partial least squares. Neuroimage 3: 143-157.

McIntosh AR, Gonzalez-Lima F (1998). Large-scale functional connectivity in associative learning: interrelations of the rat auditory, visual, and limbic systems. J Neurophysiol 80: 3148-3162.

Morecraft RJ, Van Hoesen GW (1992). Cingulate input to the primary and supplementary motor cortices in the rhesus monkey: evidence for somatotopy in areas $24 \mathrm{c}$ and $23 \mathrm{c}$. J Comp Neurol 322: 471-489.

Morecraft RJ, Van Hoesen GW (1993). Frontal granular cortex input to the cingulate (M3), supplementary (M2) and primary (M1) motor cortices in the rhesus monkey. J Comp Neurol 337: 669-689.

Mosimann UP, Rihs TA, Engeler J, Fisch H, Schlaepfer TE (2000). Mood effects of repetitive transcranial magnetic stimulation of left prefrontal cortex in healthy volunteers. Psychiatry Res 94: 251-256.

Mottaghy FM, Keller CE, Gangitano M, Ly J, Thall M, Parker JA et al (2002). Correlation of cerebral blood flow and treatment effects of repetitive transcranial magnetic stimulation in depressed patients. Psychiatry Res 115: 1-14.

Muller-Preuss P, Jurgens U (1976). Projections from the 'cingular' vocalization area in the squirrel monkey. Brain Res 103: 29-43.

Nadeau SE, McCoy KJ, Crucian GP, Greer RA, Rossi F, Bowers D et al (2002). Cerebral blood flow changes in depressed patients after treatment with repetitive transcranial magnetic stimulation: evidence of individual variability. Neuropsychiatry Neuropsychol Behav Neurol 15: 159-175.

Nahas Z, Teneback CC, Kozel A, Speer AM, DeBrux C, Molloy M et al (2001). Brain effects of TMS delivered over prefrontal cortex in depressed adults: role of stimulation frequency and coilcortex distance. J Neuropsychiatry Clin Neurosci 13: 459-470.

Ongur D, Price JL (2000). The organization of networks within the orbital and medial prefrontal cortex of rats, monkeys and humans. Cereb Cortex 10: 206-219.
Padberg F, Juckel G, Prassl A, Zwanzger P, Mavrogiorgou P, Hegerl U et al (2001). Prefrontal cortex modulation of mood and emotionally induced facial expressions: a transcranial magnetic stimulation study. J Neuropsychiatry Clin Neurosci 13: 206-212.

Pascual-Leone A, Catala MD, Pascual-Leone Pascual A (1996). Lateralized effect of rapid-rate transcranial magnetic stimulation of the prefrontal cortex on mood. Neurology 46: 499-502.

Paus T (2001). Primate anterior cingulate cortex: where motor control, drive and cognition interface. Nat Rev Neurosci 2: 417-424.

Paus T (2002). Combination of transcranial magnetic stimulation with brain imaging. In: Mazziotta J, Toga A (eds). Brain Mapping: The Methods, 2nd edn. Academic Press: New York. pp 691-705.

Paus T, Castro-Alamancos MA, Petrides M (2001). Cortico-cortical connectivity of the human mid-dorsolateral frontal cortex and its modulation by repetitive transcranial magnetic stimulation. Eur J Neurosci 14: 1405-1411.

Paus T, Jech R, Thompson CJ, Comeau R, Peters T, Evans AC (1997). Transcranial magnetic stimulation during positron emission tomography: a new method for studying connectivity of the human cerebral cortex. J Neurosci 17: 3178-3184.

Paus T, Wolforth M (1998). Transcranial magnetic stimulation during PET: reaching and verifying the target site. Hum Brain Mapp 6: 399-402.

Petrides M, Alivisatos B, Meyer E, Evans AC (1993). Functional activation of the human frontal cortex during the performance of verbal working memory tasks. Proc Natl Acad Sci USA 90: 878-882.

Petrides M, Pandya DN (1999). Dorsolateral prefrontal cortex: comparative cytoarchitectonic analysis in the human and the macaque brain and corticocortical connection patterns. Eur J Neurosci 11: 1011-1036.

Petrides M, Frey S, Chen J-K (2001). Increased activation of the mid-dorsolateral frontal cortex during the monitoring of abstract visual and verbal stimuli. Int Conf Funct Mapping Hum Brain 7: S721.

Picard N, Strick PL (1996). Motor areas of the medial wall: a review of their location and functional activation. Cereb Cortex 6: 342-353.

Pizzagalli D, Pascual-Marqui RD, Nitschke JB, Oakes TR, Larson $\mathrm{CL}$, Abercrombie $\mathrm{HC}$ et al (2001). Anterior cingulate activity as a predictor of degree of treatment response in major depression: evidence from brain electrical tomography analysis. $A m J$ Psychiatry 158: 405-415.

Post RM, Kimbrell TA, McCann UD, Dunn RT, Osuch EA, Speer AM et al (1999). Repetitive transcranial magnetic stimulation as a neuropsychiatric tool: present status and future potential. J ECT 15: 39-59.

Ryan RM, Frederick C (1997). On energy, personality, and health: subjective vitality as a dynamic reflection of well-being. J Pers 65: 529-565.

Shajahan PM, Glabus MF, Steele JD, Doris AB, Anderson K, Jenkins JA et al (2002). Left dorso-lateral repetitive transcranial magnetic stimulation affects cortical excitability and functional connectivity, but does not impair cognition in major depression. Prog Neuropsychopharmacol Biol Psychiatry 26: 945-954.

Speer AM, Kimbrell TA, Wassermann EM, Repella D, Willis MW, Herscovitch $\mathrm{P}$ et al (2000). Opposite effects of high and low frequency rTMS on regional brain activity in depressed patients. Biol Psychiatry 48: 1133-1141.

Speer AM, Willis MW, Herscovitch P, Daube-Witherspoon M, Shelton JR, Benson BE et al (2003). Intensity-dependent regional cerebral blood flow during $1-\mathrm{Hz}$ repetitive transcranial magnetic stimulation (rTMS) in healthy volunteers studied with $\mathrm{H} 215 \mathrm{O}$ positron emission tomography: II. Effects of prefrontal cortex rTMS. Biol Psychiatry 54: 826-832. 
Strafella AP, Paus T, Barrett J, Dagher A (2001). Repetitive transcranial magnetic stimulation of the human prefrontal cortex induces dopamine release in the caudate nucleus. J Neurosci 21: RC157.

Sutton D, Larson C, Lindeman RC (1974). Neocortical and limbic lesion effects on primate phonation. Brain Res 71: 61-75.

Talairach J, Tournoux P Co-Planar (1988). Stereotaxic Atlas of the Human Brain. Thieme Medical Publishers: New York.

Teneback CC, Nahas Z, Speer AM, Molloy M, Stallings LE, Spicer KM et al (1999). Changes in prefrontal cortex and paralimbic activity in depression following two weeks of daily left prefrontal TMS. J Neuropsychiatry Clin Neurosci 11: 426-435.

Videbech P, Ravnkilde B, Pedersen TH, Hartvig H, Egander A, Clemmensen K et al (2002). The Danish PET/depression project: clinical symptoms and cerebral blood flow. A regions-of-interest analysis. Acta Psychiatr Scand 106: 35-44.

Watson D, Clark LA, Tellegen A (1988). Development and validation of brief measures of positive and negative affect: the PANAS scales. J Pers Soc Psychol 54: 1063-1070.

Woods RP, Mazziotta JC, Cherry SR (1993). MRI-PET registration with automated algorithm. J Comput Assist Tomogr 17: 536-546.

Worsley KJ, Evans AC, Marrett S, Neelin P (1992). A threedimensional statistical analysis for $\mathrm{CBF}$ activation studies in human brain. J Cereb Blood Flow Metab 12: 900-918.

Vogt BA, Nimchinsky EA, Vogt LJ, Hof PR (1995). Human cingulate cortex: surface features, flat maps and cytoarchitecture. J Comp Neurol 359: 490-506. 